\title{
A First-Principles Study on Na and O Adsorption Behaviors on Mo (110) Surface
}

\author{
Qingqing Zeng ${ }^{1}$, Zhixiao Liu ${ }^{1, *}$, Wenfeng Liang ${ }^{2}$, Mingyang Ma ${ }^{2, *}$ and Huiqiu Deng ${ }^{3}$ \\ 1 College of Materials Science and Engineering, Hunan University, Changsha 410082, China; \\ Qqzeng@hnu.edu.cn \\ 2 Institute of Nuclear Physics and Chemistry, China Academy of Engineering Physics, Mianyang 621900, China; \\ liangwf@caep.cn \\ 3 School of Physics and Electronics, Hunan University, Changsha 410082, China; hqdeng@hnu.edu.cn \\ * Correspondence: zxliu@hnu.edu.cn (Z.L.); mamingyang@caep.cn (M.M.)
}

Citation: Zeng, Q.; Liu, Z.; Liang, W.; Ma, M.; Deng, H. A First-Principles Study on $\mathrm{Na}$ and $\mathrm{O}$ Adsorption Behaviors on Mo (110) Surface. Metals 2021, 11, 1322. https://doi.org/ $10.3390 /$ met11081322

Academic Editor: Guy Makov

Received: 16 July 2021

Accepted: 16 August 2021

Published: 20 August 2021

Publisher's Note: MDPI stays neutral with regard to jurisdictional claims in published maps and institutional affiliations.

Copyright: (c) 2021 by the authors. Licensee MDPI, Basel, Switzerland. This article is an open access article distributed under the terms and conditions of the Creative Commons Attribution (CC BY) license (https:// creativecommons.org/licenses/by/ $4.0 /)$.

\begin{abstract}
Molybdenum-rhenium alloys are usually used as the wall materials for high-temperature heat pipes using liquid sodium as heat-transfer medium. The corrosion of Mo in liquid Na is a key challenge for heat pipes. In addition, oxygen impurity also plays an important role in affecting the alloy resistance to $\mathrm{Na}$ liquid. In this article, the adsorption and diffusion behaviors of $\mathrm{Na}$ atom on Mo (110) surface are theoretically studied using first-principles approach, and the effects of alloy $\mathrm{Re}$ and impurity $\mathrm{O}$ atoms are investigated. The result shows that the Re alloy atom can strengthen the attractive interactions between $\mathrm{Na} / \mathrm{O}$ and the Mo substrate, and the existence of $\mathrm{Na}$ or $\mathrm{O}$ atom on the Mo surface can slower down the Na diffusion by increasing diffusion barrier. The surface vacancy formation energy is also calculated. For the Mo (110) surface, the $\mathrm{Na} / \mathrm{O}$ co-adsorption can lead to a low vacancy formation energy of $0.47 \mathrm{eV}$, which indicates the dissolution of Mo is a potential corrosion mechanism in the liquid $\mathrm{Na}$ environment with $\mathrm{O}$ impurities. It is worth noting that $\mathrm{Re}$ substitution atom can protect the Mo surface by increasing the vacancy formation energy to $1.06 \mathrm{eV}$.
\end{abstract}

Keywords: Mo-Re alloy; Na adsorption and diffusion; surface vacancy; first-principles calculation

\section{Introduction}

Alkali metal heat pipes (HPs) are initially designed for heat transfer in space nuclear power systems, of which the operating temperature is typically from $800 \mathrm{~K}$ to $1800 \mathrm{~K}$. HPs using alkali metals are also promising in advanced energy and power systems such as highefficiency waste heat utilization [1], hypersonic vehicles [2], and molten salt reactors [3].

A heat pipe consists of a sealed shell, wick structure and a vapor chamber containing working fluid, which is normally filled after the shell is evacuated [4]. Heat transfer in a heat pipe is achieved passively by the phase change and the circulation of the working fluid [5]. Different types of working fluid and shell material are adopted in heat pipes used under different working conditions. The type of heat pipe can be divided into four main types according to their working temperature: low temperature heat pipe $\left(-270 \sim 0{ }^{\circ} \mathrm{C}\right)$, normal temperature heat pipe $\left(0 \sim 200{ }^{\circ} \mathrm{C}\right)$, medium temperature heat pipe $\left(200 \sim 600{ }^{\circ} \mathrm{C}\right)$ and high temperature heat pipe (above $600^{\circ} \mathrm{C}$ ) [6].

Alkali metal heat pipes operating at temperature above $800 \mathrm{~K}$ have typically been constructed taking liquid alkali metal: potassium, sodium, or lithium as the working fluid due to their high-power capacity and great thermal stability [7]. For alkali metal heat pipes used in nuclear power systems, a key requirement is the compatibility of structure materials with both nuclear fuel and alkali metal [8]. Refractory metals and alloys, for owning both high creep strength at high temperatures and excellent compatibility with alkali liquid metals as well as nuclear fuel, are often applied as the shell material of alkali metal heat pipes [9]. Molybdenum (Mo) is a kind of refractory metal that is reported to be one of the greatest candidates for use of alkali metal heat pipe walls [10-13]. Recently, Mo 
alloys are also considered as structural materials using in nuclear reactors [14,15]. However, pure Mo becomes brittle at about room temperature and below, which impacts heavily not only on the fabrication of heat pipes, but also the transportation process that easily leading to heat pipe breakage $[16,17]$. To tackle this problem, adding rhenium $(\mathrm{Re})$ into pure molybdenum is found to be effective in enhancing low temperature ductility while also improving the high-temperature strength and creep resistance, known as the "rhenium effect" $[18,19]$. Mo-Re alloy has great advantage in high temperature heat pipes, where the operating environment is usually highly oxidizing and corrosive.

One issue for Mo-Re alloy in the heat pipe is the corrosion induced by the liquid metal working fluid and impurities. Studies have shown that dissolution, mass transfer and impurity reactions are the major corrosion mechanisms in refractory metal-alkali systems. Meanwhile, the most serious corrosion problems encountered are related to impurity reactions associated with oxygen [20]. Even though the addition of Re can improve the low temperature ductility, creep resistance and high temperature strength, it is not known that whether the Re atom could bring an improvement on the corrosion resistance of Mo. In addition, as the existence of oxygen $(\mathrm{O})$ could lead to serious corrosion problems, it is important to learn the chemical interaction between $\mathrm{O}$ and Mo surface [21]. In this study, we used a first-principles approach to investigate basic properties such as adsorption, diffusion properties of $\mathrm{Na}$ atom and $\mathrm{O}$ atom on the pure Mo (110) surface and Mo-Re (110) surface. In addition, the formation of surface vacancy was calculated for evaluating if the adsorbates can prevent Mo from dissolution.

\section{Computational Methods and Model}

We employed the Vienna Ab-initio Simulation Package (VASP, version 5.4, developed by Vienna University) [22,23] to carry out our first-principles calculations. All calculations were implemented based on density-functional theory (DFT) $[24,25]$, using a plane wave basis set [26]. The interactions between the core and valence electrons were described with the projector augmented wave (PAW) approach $[27,28]$. Generalized gradient approximation (GGA) of the Perdew-Burke-Ernzerhof (PBE) functional [29] was adopted in all the calculations. An energy cut-off for the plane-wave basis set was set to $380 \mathrm{eV}$ for both the relaxed and static computations. The residual force for structure optimization was less than $0.02 \mathrm{eV} / \AA$. The $7 \times 7 \times 1$ k-point grids generated by the Monkhorst-Pack (MP) technique [30] was found to be sufficient for the present study.

The Mo (110) surface was used to explore the interaction between $\mathrm{Na} / \mathrm{O}$ atoms and the Mo substrate because the Mo (110) surface has the lowest surface energy as reported in Materials Project Database [31]. Mo (110)- $(2 \times 2)$ surface cell was represented by a 5-layers slab model (8 atoms per layer) with the vacuum thickness of $10 \AA$. The upper two layers were fully relaxed and the bottom three layers were fixed as the bulk phase during structure optimization. In this study, we also investigated the effect of alloy element Re on the adsorption of $\mathrm{Na}$ and $\mathrm{O}$ atoms. For Mo-Re alloy, when the weight percentage of Re is less than $14 \%$, Re atoms randomly replace Mo atoms at the lattice site [32]. We considered three adsorption sites for the $\mathrm{Na}$ and $\mathrm{O}$ atom on Mo/Mo-Re alloy: TOP site, Bridge site and Hollow site of Mo/Mo-Re (110) surface. Top site is located over the Mo atoms of the topmost layer, and bridge site is located between the two Mo atoms of the topmost layer, while Hollow site is above the Mo atoms of the second layer, as is shown in Figure 1. For one atom adsorbed on the (110)- $(2 \times 1)$ surface cell, the corresponding coverage $(\Theta)$ is $\frac{1}{8}$ ML.

The climbing image nudged-elastic-band (CI-NEB) method was employed to calculate the $\mathrm{Na}$ diffusion barriers between two most stable adsorption sites on $\mathrm{Mo} / \mathrm{Mo}-\mathrm{Re}$ (110) surface [33]. 
(a)

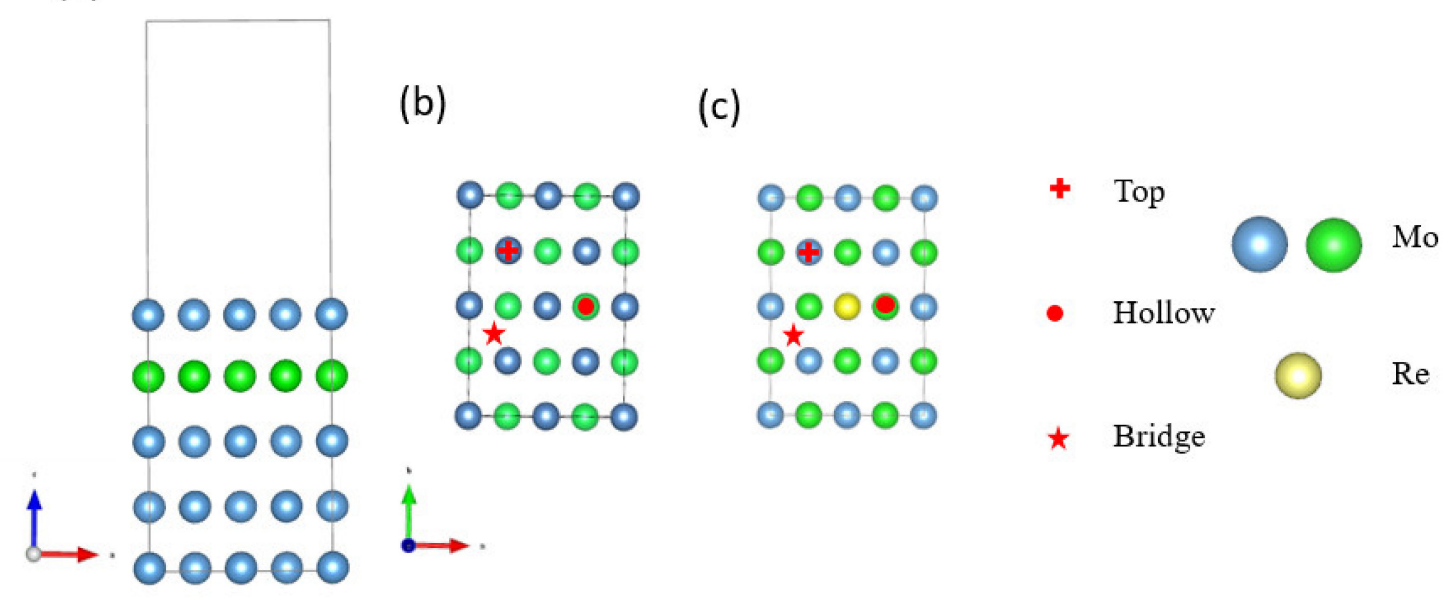

Figure 1. Structure of Mo/Mo-Re (110) surfaces and adsorption sites: (a) side view of Mo/Mo-Re (110) slab model; (b) Top view of Mo (110) surface and (c) top view of Mo-Re surface. Blue and green spheres represent Mo atoms, and yellow spheres represent Re atoms.

\section{Results and Discussion}

\subsection{Model Verification}

Molybdenum (Mo) has a body centered cubic (bcc) structure with an experimental lattice constant of $3.14 \AA$ [34] and each conventional cell has 2 atoms. The theoretical lattice constant found in this study is $3.15 \AA$ which agrees well with the experimental value.

In order to find out the thermodynamically most stable surface structure of bcc-Mo, the surface energy is calculated by the following equation:

$$
\sigma=\left(E_{\text {slab }}-\mathrm{n} \times \mathrm{E}_{\text {bulk }}\right) /(2 \mathrm{~A})
$$

where $\mathrm{A}$ is the surface area and $\mathrm{n}$ is the number of atoms in the slab model, $E_{\text {slab }}$ is the total energy of the surface, $\mathrm{E}_{\text {bulk }}$ is the energy per Mo atom of the ground state structure.

The surface energies of low-index Mo surfaces are shown in Table 1. The surface energies of Mo (100), Mo (110) and Mo (111) are $0.22 \mathrm{eV} / \AA^{2}, 0.17 \mathrm{eV} / \AA^{2}$ and $0.18 \mathrm{eV} / \AA^{2}$, respectively. Our results are the same with date reported in Materials Project Database [31]. All above results demonstrate that the input parameters and surface models used in the present study are reliable. In addition, considering that Mo (110) surface has the lowest surface energy, we focus on understanding $\mathrm{Na}$ and $\mathrm{O}$ adsorption properties on this energetically most stable surface.

Table 1. Surface energies of low-index surfaces.

\begin{tabular}{ccc}
\hline & \multicolumn{2}{c}{ Surface Energy(eV/ $\left.\AA^{\mathbf{2}}\right)$} \\
\hline Surface & Present Calculation & Materials Project Database \\
$(100)$ & 0.22 & 0.22 \\
$(110)$ & 0.17 & 0.17 \\
$(111)$ & 0.18 & 0.18 \\
\hline
\end{tabular}

\subsection{Na Atom and O Atom Adsorption}

The adsorption behaviors of Sodium atom on both Mo (110) and Mo-Re (110) surfaces are investigated firstly. In the current calculations, the adsorption energy for the Na atom adsorbed on the Mo (110) or Mo-Re (110) surfaces is defined as:

$$
E_{a d s}=E_{(M o / M o-R e)-N a}-E_{(M o / M o-R e) s l a b}-E_{N a}
$$


Here $E_{(\mathrm{Mo} / \mathrm{Mo}-\mathrm{Re})-\mathrm{Na}}$ is the total energy of the adsorbate-substrate system, $E_{(\mathrm{Mo} / \mathrm{Mo}-\mathrm{Re}) \text { slab }}$ is the total energy of the clean Mo/Mo-Re surface, and $E_{\mathrm{Na}}$ is the average energy of BCC Na. A negative $E_{a d s}$ value indicates the attractive interaction between the adsorbate and the substrate. The more negative adsorption energy implies the stronger attractive interaction between the adsorbate and the substrate.

Figure 2 shows the Initial states and final states of $\mathrm{Na}$ adsorption Mo (110) and MoRe (110) surfaces. The adsorption energies and structural parameters of $\mathrm{Na}$ on Mo or Mo-Re (110) surfaces are given in Table 2. For the Mo (110) surface, the hollow site is the energetically most favored for $\mathrm{Na}$ adsorption and the corresponding energy is $-0.51 \mathrm{eV}$. The Bridge site is second preferred for $\mathrm{Na}$ adsorption with the adsorption energy of $-0.44 \mathrm{eV}$. The Top site is a stable one for $\mathrm{Na}$ adsorption, but the adsorption energy is only $-0.35 \mathrm{eV}$. For Mo-Re (110) surface, all un-equivalent Top, Hollow and Bridge sites are considered for $\mathrm{Na}$ adsorption. For each type of adsorption site, only the initial and final sites with the lowest energy are collected and shown in Figure 2 and Table 2. Compared with the pristine Mo (110) surface, Mo-Re (110) surface provides stronger affinity to the Na atom. It is worth noting that the $\mathrm{Na}$ atom initially placed at the Bridge site moves towards the Re atom after the structure optimization, as can be seen in Figure $2 \mathrm{f}$, and this configuration delivers the lowest adsorption energy of $-0.60 \mathrm{eV}$. The adsorption energies of $\mathrm{Na}$ adsorption on the Top site and Hollow site of Mo-Re (110) surface are $-0.43 \mathrm{eV}$ and $-0.56 \mathrm{eV}$, respectively. The average vertical distance between adsorbed $\mathrm{Na}$ atom and the top layer $\left(d_{\mathrm{Na}-\mathrm{sur}}\right)$ is also listed in Table 2. It is interesting to found that the $\mathrm{Na}$ adsorption energy is proportional to $d_{\mathrm{Na}-\text { surf }}$ (Figure 3). A shorter distance between the $\mathrm{Na}$ atom and the top layer indicates a stronger attractive interaction. Both energetical and geometric parameters indicate that the Re atom can strengthen the attractive interaction between $\mathrm{Na}$ and the Mo surface. The electronic structure is analyzed for understanding the effect of Re atom on Na adsorption. On the Mo (110) surface, the Na atom only interacts with Mo atoms in the upmost layer, as shown in Figure 4a. The Re atom can significantly affect the charge redistribution induced a by $\mathrm{Na}$ adsorption. As shown in Figure $4 \mathrm{~b}$, electrons from the Mo settled in the second atomic layer migrate to the first layer, resulting to great electron accumulation. In this case, the columbic attraction between the $\mathrm{Na}$ atom and the substrate is enhanced and lead to a lower adsorption energy.

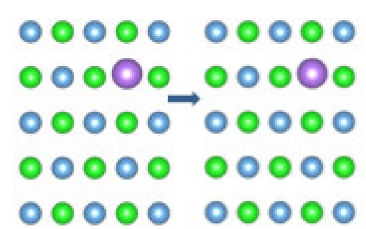

(a) Top

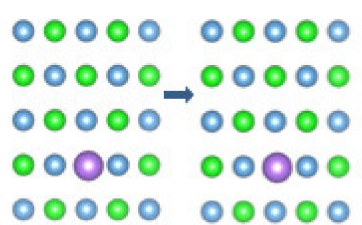

(b) Hollow

Pristine Mo (110) surface

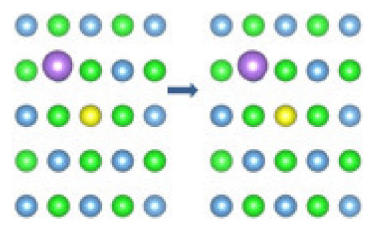

(d) Top

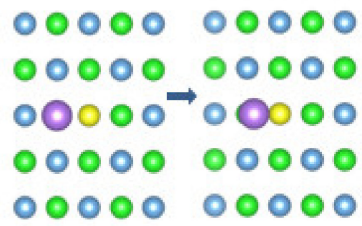

(e) Hollow

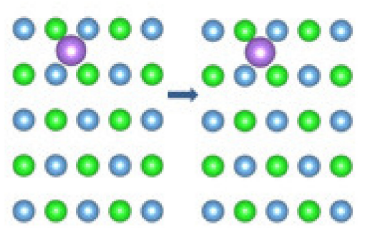

(c) Bridge

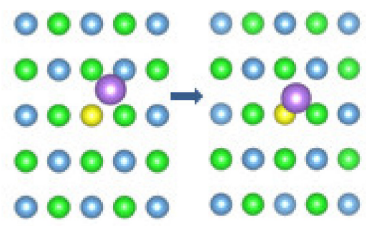

(f) Bridge

Mo-Re (110) surface

Figure 2. Initial and final configurations of Na adsorption on Mo (110) and Mo-Re (110) surfaces. Snaps (a-c) show Na atoms initially placed at Top site, Hollow site and Bridge site of Mo (110) surface. Snaps (d-f) show Na atoms initially placed at Top site, Hollow site and Bridge site of Mo-Re (110) surface. Blue and green spheres represent Mo atoms in the first layer and the second layer. Yellow and purple spheres represent Re atoms and $\mathrm{Na}$ atoms, respectively. 
Table 2. Adsorption energy of $\mathrm{Na}$ on $\mathrm{Mo}(110)$ and Mo-Re (110) surfaces. $E_{a d s}$ is the adsorption energy, and $d_{N a-s u r f}$ is the vertical distance between the adsorbate and the top layer of the slab model.

\begin{tabular}{ccccc}
\hline Surface & Initial Site & $\boldsymbol{d}_{\boldsymbol{N a}-\text { sur }}(\AA)$ & $\boldsymbol{E}_{\text {ads }}(\mathbf{e V})$ & Final Site \\
\hline \multirow{3}{*}{ Mo (110) } & Top & 2.92 & -0.35 & Top \\
& Hollow & 2.74 & -0.51 & Hollow \\
& Bridge & 2.82 & -0.44 & Bridge \\
\hline \multirow{3}{*}{ Mo-Re (110) } & Top & 2.82 & -0.43 & Top \\
& Hollow & 2.71 & -0.56 & Hollow \\
& Bridge & 2.68 & -0.60 & Bridge \\
\hline
\end{tabular}

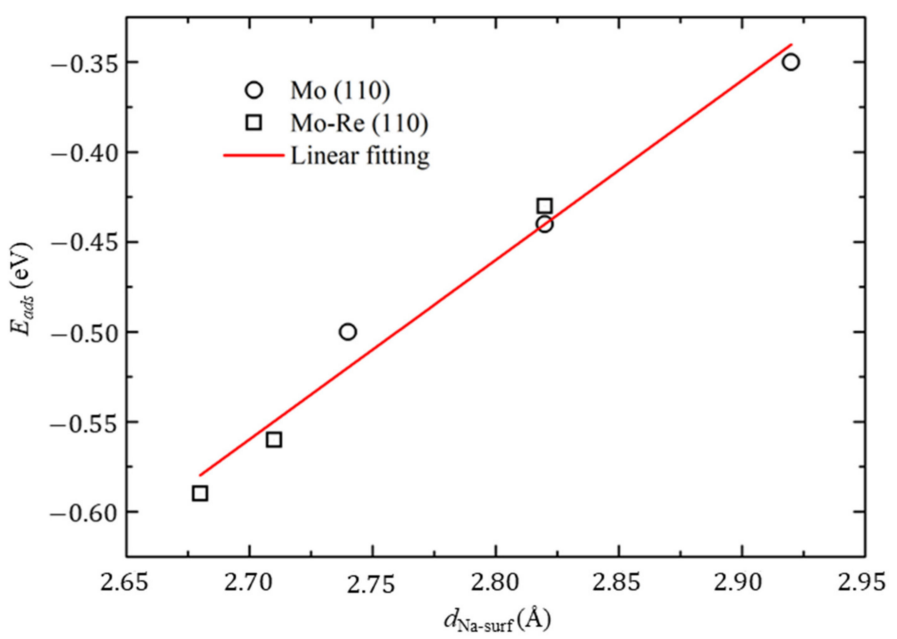

Figure 3. Na adsorption energy as the function of the average distance between the Na atom and the top layer of the substrate.
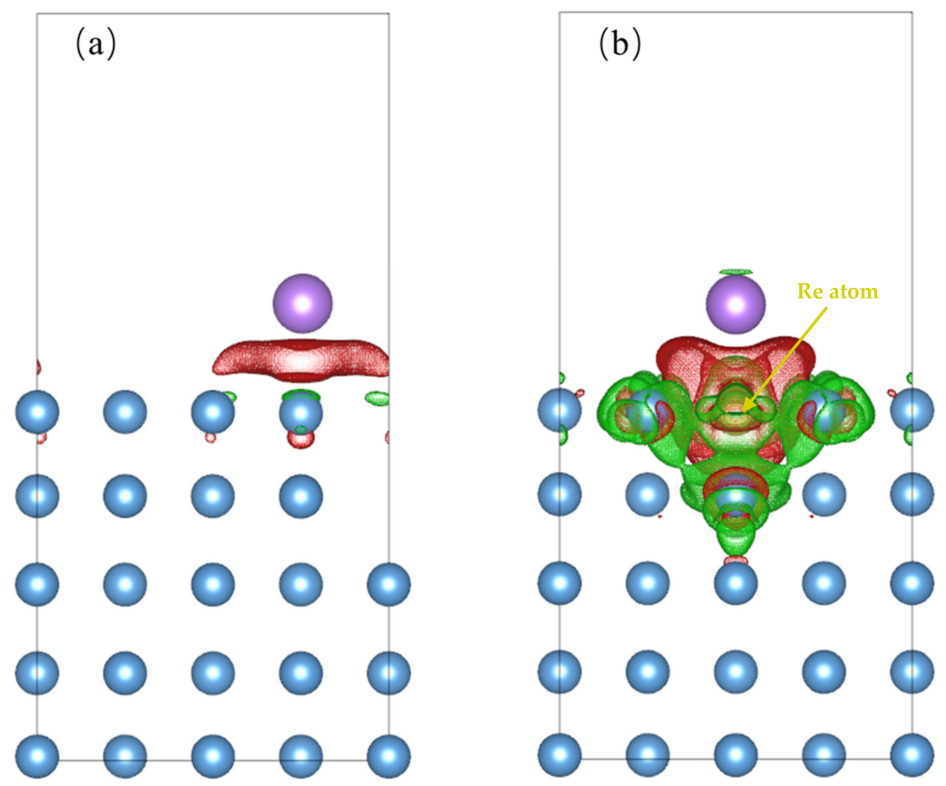

Figure 4. Difference charge density of $\mathrm{Na}$ adsorption at the hollow site of (a) Mo (110) surface and (b) Mo-Re (110) surface. The red isosurface represent the electron accumulation region, while the green isosurface represent the electron depletion region. The Re atom is located in the center of Mo (110) surface, as shown in Figure 1c. 
The adsorption energies of $\mathrm{O}$ on Mo and Mo-Re (110) surfaces are also investigated in the present study (Table 3). The adsorption energy for the $\mathrm{O}$ atom adsorbed on the $\mathrm{Mo} / \mathrm{Mo}-\operatorname{Re}(110)$ surfaces is defined as

$$
E_{a d s}=E_{(M o / M o-R e)-O}-E_{(M o / M o-R e) s l a b}-\frac{1}{2} E_{O_{2}}
$$

where $E_{(\mathrm{Mo} / \mathrm{Mo}-\mathrm{Re})-\mathrm{O}}$ is the total energy of the adsorbate-substrate system, $E_{(\mathrm{Mo} / \mathrm{Mo}-\mathrm{Re}) \text { slab }}$ is the total energy of clean Mo/Mo-Re surfaces, and $E_{\mathrm{O}_{2}}$ is the energy of an isolated $\mathrm{O}_{2}$ molecule. The adsorption energy of a single $\mathrm{O}$ atom on the surface is always calculated to characterize the oxygen-substrate interactions for refractory materials, and the energy of an $\mathrm{O}$ atom is usually referenced to the half of the $\mathrm{O}_{2}$ molecule [35-37].

Table 3. Adsorption energy of O on Mo/Mo-Re (110) surface.

\begin{tabular}{ccccc}
\hline Surface & Initial Site & $\boldsymbol{d}_{\boldsymbol{O}-\text { surf }}(\AA)$ & $\boldsymbol{E}_{\text {ads }}(\mathbf{( e V})$ & Final Site \\
\hline \multirow{3}{*}{ Mo (110) } & Top & 2.18 & -2.80 & Top \\
& Hollow & 1.15 & -4.09 & Hollow \\
& Bridge & 1.15 & -4.09 & Hollow \\
\hline \multirow{3}{*}{ Mo-Re (110) } & Top & 1.73 & -2.82 & Top \\
& Hollow & 1.14 & -4.14 & Hollow \\
& Bridge & 1.14 & -4.14 & Hollow \\
\hline
\end{tabular}

As listed in Table 3, The Hollow site is energetically preferred for O adsorption on the Mo (110) surface with the adsorption energy of $-4.09 \mathrm{eV}$. It is worth noting that the $\mathrm{O}$ atom initially placed at the Bridge site will spontaneously move to the Hollow site after the structure optimization as shown in Figure 5c. The $\mathrm{O}$ atom can also be stabilized at the Top site, but the adsorption energy is only $-2.80 \mathrm{eV}$. As with Na on Mo (110) surface, the shorter vertical distance between $\mathrm{O}$ atom and the substrate $\left(d_{\mathrm{O}-\text { surf }}\right)$ leads to a lower (more negative) adsorption energy. For the Mo-Re (110) surface, the Hollow site is also the most favored for $\mathrm{O}$ adsorption and the corresponding adsorption energy is $-4.14 \mathrm{eV}$, which is even 0.05 lower than the adsorption energy of $\mathrm{O}$ at the Hollow site of Mo (110) surface. It is worth mentioning that all un-equivalent Hollow sites around the Re atoms are checked, and Figure 5 as well as Table 3 demonstrates configurations with the lowest energy. As with the pristine Mo (110) surface, the $\mathrm{O}$ atom initially placed at the Bridge site of the Mo-Re (110) surface will move to the Hollow site after the structure relaxation. In addition, the $\mathrm{O}$ atom can be stabilized at the Top site with a much higher adsorption energy of $-2.82 \mathrm{eV}$. As with $\mathrm{Na}$ adsorption, the Re atom in the surface can also strengthen the attractive interaction between the adsorbed $\mathrm{O}$ atom and the Mo-based substrate. 


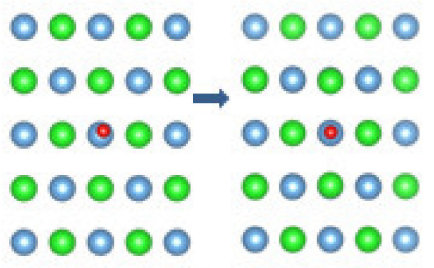

(a) Top

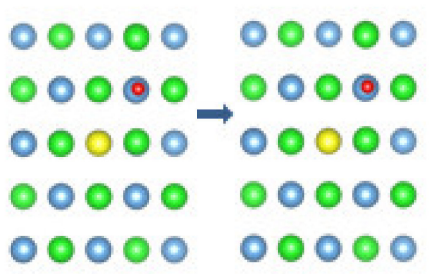

(d) Top

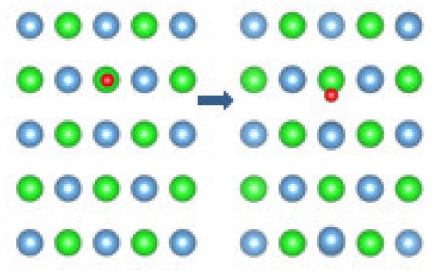

(b) Hollow

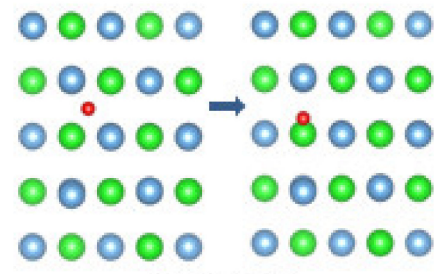

(c) Bridge

Prestine Mo (110) surface

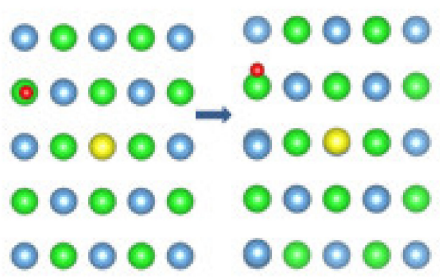

(e) Hollow

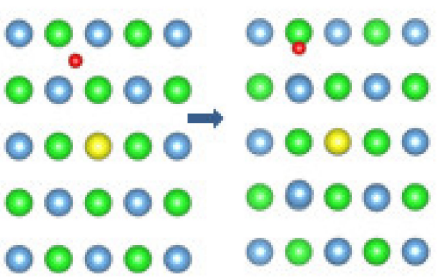

(f) Bridge

Mo-Re (110) surface

Figure 5. Initial and final configurations of $\mathrm{O}$ adsorption on Mo (110) and Mo-Re (110) surfaces. Scheme 110. surface. Snaps $(\mathbf{a}-\mathbf{c})$ show $\mathrm{O}$ atoms initially placed at Top site, Hollow site and Bridge site of Mo (110) surface, while snaps (d-f) show $\mathrm{O}$ atoms initially placed at Top site, Hollow site and Bridge site of Mo-Re (110) surface. Blue and green spheres represent Mo atoms in the first layer and the second layer. Yellow and red spheres represent Re atoms and $\mathrm{O}$ atoms, respectively.

\subsection{Impact of $\mathrm{O}$ on $\mathrm{Na}$ Adsorption and Diffusion}

$\mathrm{O}$ is the key impurity in liquid metal for the high-temperature heat pipe. The impact of pre-adsorbed $\mathrm{O}$ on $\mathrm{Na}$ adsorption behavior is also investigated. The configurations of pre-adsorbed $\mathrm{O}$ atom are adopted from Figure $6 \mathrm{~b}$,e. The adsorption energy for the Na on surface with a pre-adsorbed $\mathrm{O}$ is defined as

$$
E_{a d s}=E_{(M o-O / M o-R e-O)-N a}-E_{(M o-O / M o-R e-O) s l a b}-E_{N a}
$$

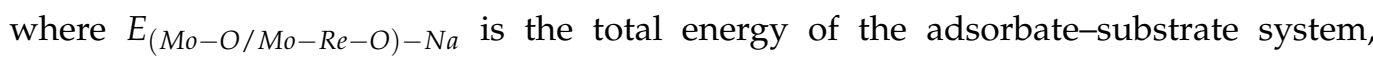
$E_{(M o-O / M o-R e-O) s l a b}$ is the total energy of Mo or Mo-Re surface with a pre-adsorbed $\mathrm{O}$ atom, and $E_{\mathrm{Na}}$ is the energy of an Na atom in the BCC structure. In Equation (4), the subscript Mo-O represents the Mo (110) surface with a pre-adsorbed $\mathrm{O}$ atom and Mo-Re-O represent the Mo-Re (110) surface with a pre-adsorbed O atom.

All un-equivalent sites are considered and only the configurations with the lowest energies are shown in Figure 6. Adsorption energies and geometric parameters are given in Table 4. It is found that Na atoms initially placed at Top and Bridge sites move to Hollow sites after the optimization. The former one occupied the Hollow site which is $6.02 \AA$ away from the pre-adsorbed $\mathrm{O}$ atom, and latter one occupied the Hollow site which is only $2.35 \AA$ away from the $\mathrm{O}$ atom. However, these two final configurations lead to the same adsorption energy of $0.52 \mathrm{eV}$. For the $\mathrm{Na}$ atom initially placed at the Hollow site, the adsorption energy is $-0.53 \mathrm{eV}$ with $d_{\mathrm{Na}-\mathrm{O}}=2.36 \AA$. It can be inferred that the $\mathrm{O}$ atom does not affect the adsorption behavior of the $\mathrm{Na}$ atom. 


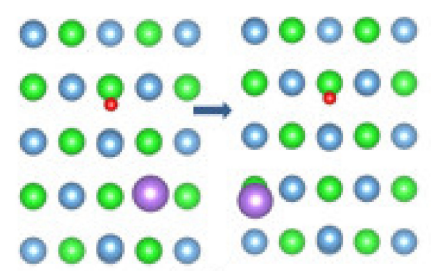

(a) Top

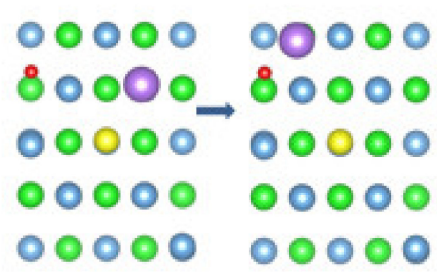

(d) Top

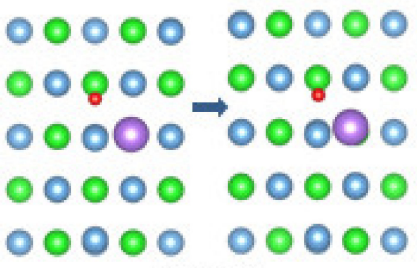

(b) Hollow

Mo-O (110) surface

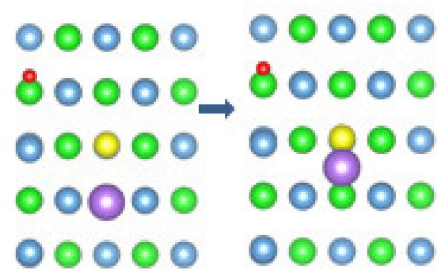

(e) Hollow

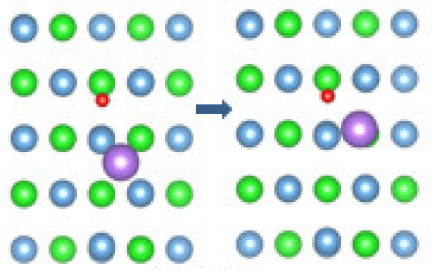

(c) Bridge

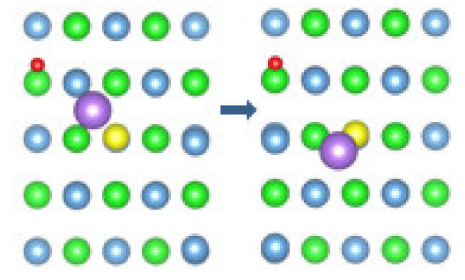

(f) Bridge

Mo-Re-O (110) surface

Figure 6. The initial and final configurations of Na atom adsorption on Mo-O (110) and Mo-Re-O (110) surface. Snaps (a-c) show Na atoms initially placed at Top site, Hollow site and Bridge site of Mo-O (110) surface, while snaps (d-f) show $\mathrm{Na}$ atoms initially placed at Top site, Hollow site and Bridge site of Mo-Re-O (110) surface. Blue and green spheres represent Mo atoms in the first layer and the second layer. Yellow, purple and red spheres represent $\mathrm{Re}$ atoms, $\mathrm{Na}$ atoms and $\mathrm{O}$ atoms, respectively.

Table 4. Na adsorption energy on different surface models.

\begin{tabular}{cccccc}
\hline Surface & Initial Site & $\boldsymbol{d}_{\mathbf{N a}-\mathbf{O}}(\AA)$ & $\boldsymbol{d}_{\mathbf{N a}-\mathbf{s u r}}(\AA)$ & $\boldsymbol{E}_{\boldsymbol{a d s}}(\mathbf{e V})$ & Final Stie \\
\hline \multirow{3}{*}{ Mo-O (110) } & Top & 6.02 & 2.78 & -0.52 & Hollow \\
& Hollow & 2.36 & 2.68 & -0.53 & Hollow \\
& Bridge & 2.35 & 2.83 & -0.52 & Hollow \\
\hline \multirow{3}{*}{ Mo-Re-O (110) } & Top & 2.37 & 2.76 & -0.54 & Hollow \\
& Hollow & 4.77 & 2.71 & -0.61 & Hollow \\
& Bridge & 3.18 & 2.77 & -0.57 & Bridge \\
\hline
\end{tabular}

For the Mo-Re (110) surface with a pre-adsorbed $\mathrm{O}$, Na initially placed at the top site will spontaneously move to a Hollow site which is close to the $\mathrm{O}$ atom $\left(d_{\mathrm{Na}-\mathrm{O}}=2.37 \AA\right)$ as shown in Figure 6d. However, it should be noticed that the Na atom at the Hollow site which is closer to a Re atom has lowest adsorption energy of $-0.61 \mathrm{eV}$ in Figure 6e. The adsorption energy of $\mathrm{Na}$ at the Bridge site is $-0.57 \mathrm{eV}$, which is also closer to the Re atom in Figure $6 \mathrm{f}$ and has a lower adsorption energy than the Na atom shown in Figure 6d. For the Na adsorption on the clean Mo-Re (110) surface, the adsorption energies of the Hollow site and Bridge site are $-0.56 \mathrm{eV}$ and $-0.60 \mathrm{eV}$. As with the Mo (110) surface, pre-adsorbed $\mathrm{O}$ on the Mo-Re (110) surface cannot affect the Na adsorption behavior significantly.

Figure 7 show the energy barrier of Na migration from one most stable site to its first-nearest most stable site is also calculated in this work using CI-NEB method. Our theoretical results show that the diffusion barrier of Na on Mo (110) surface is $0.037 \mathrm{eV}$, while it is $0.063 \mathrm{eV}$ on the Mo-Re (110) surface. It can be inferred that the Re atom can slower down the Na diffusion kinetics on the Mo surface. The impact of pre-adsorbed O on the Na diffusion is also investigated. For the Mo (110) surface, the pre-adsorbed $\mathrm{O}$ atom can increase the diffusion barrier to 0.087; for the Mo-Re (110) surface, the pre-adsorbed O can significantly increase the Na diffusion barrier to $0.221 \mathrm{eV}$. Therefore, it can be inferred 
that both the existence of $\mathrm{O}$ impurity and Re alloy atoms can block the Na diffusion on the Mo surface.
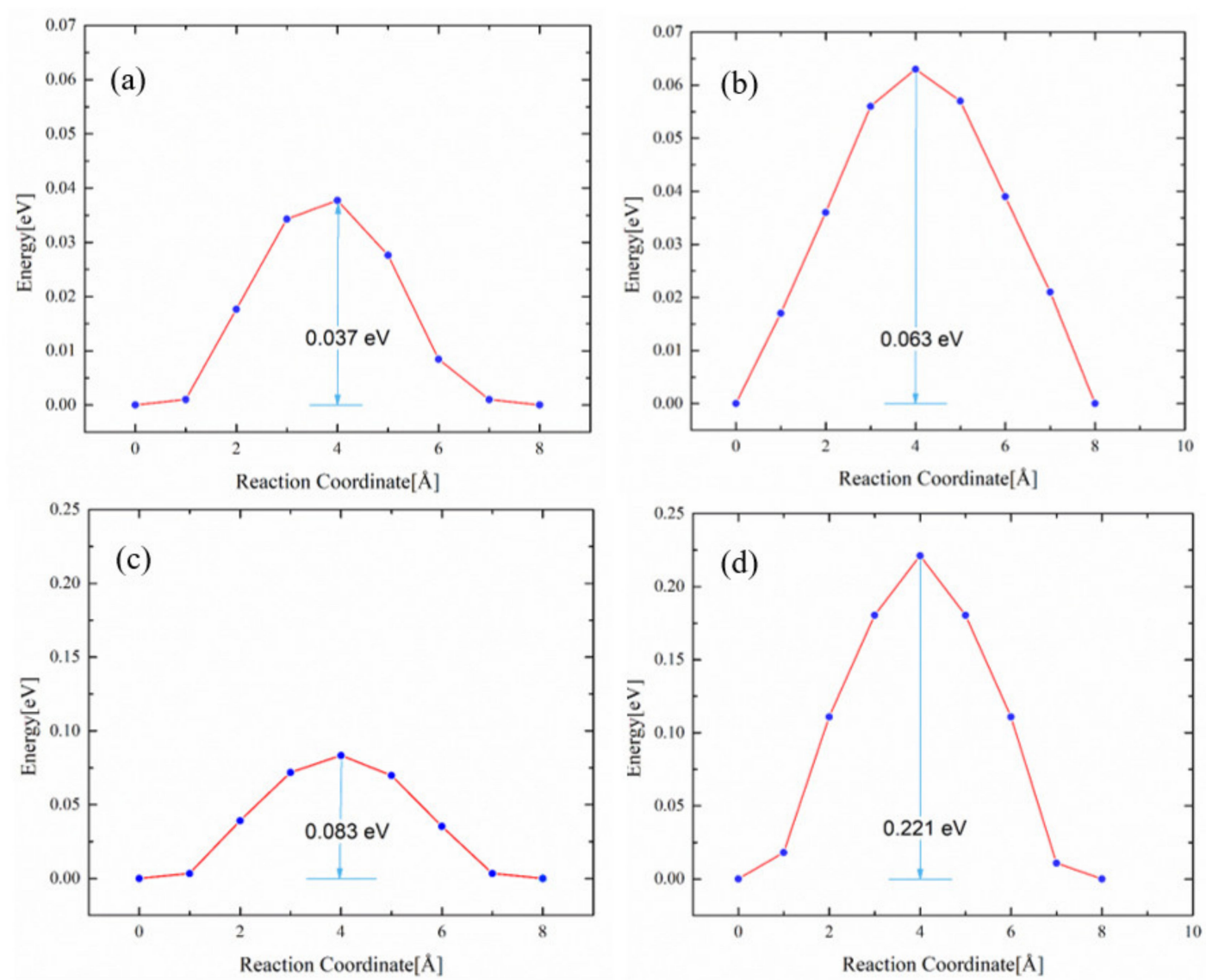

Figure 7. Calculated diffusion energy profiles for a Na atom diffusion on different surfaces: (a) Na diffusion on pure Mo (110) surface; (b) Na diffusion on Mo-Re (110) surface; (c) Na diffusion on Mo (110) surface with an adsorbed O atom; (d) Na diffusion on Mo-Re (110) surface with an adsorbed $\mathrm{O}$ atom.

3.4. Impact of $\mathrm{O} / \mathrm{Na}$ atom Adsorption on the Vacancy Formation of Mo (110) and Mo-Re (110) Surfaces

We propose that Mo dissolution into liquid $\mathrm{Na}$ is a potential scenario of Mo corrosion. In this study, the Mo vacancy formation energy is also calculated to evaluate the effect of $\mathrm{O}$ and Re on the corrosion resistance of Mo surface. The vacancy formation energy is defined using the following Equation (5):

$$
E_{\text {vac }}^{f}=E_{\text {vac-surface }}+E_{\text {Mo }}-E_{\text {surface }}
$$

where $E_{\text {surface }}$ stands for the energy of surfaces without vacancies, $E_{M o}$ is the energy of a Mo atom in its BCC structure and $E_{\text {vac-surface }}$ is the surface models with a Mo vacancy in the upmost layer.

Table 5 shows that the vacancy formation energies are $1.45 \mathrm{eV}$ and $1.32 \mathrm{eV}$ for the clean Mo (110) surface and the clean Mo-Re (110) surface, respectively. Therefore, introducing Re atom into the Mo (110) surface can enhance forming surface vacancies. The surface vacation formation energies of Mo (110) or Mo-Re (110) surface with an $\mathrm{Na}$ or O atom is also calculated. A single $\mathrm{Na}$ atom or $\mathrm{O}$ atom can reduce the surface vacancy formation energy by $0.4 \sim 0.5 \mathrm{eV}$. It can be inferred that a low coverage of $\Theta=\frac{1}{8} \mathrm{ML}$ of $\mathrm{Na}$ or $\mathrm{O}$ can destabilize the Mo (110) and Mo-Re (110) surface. The effect of Na/O synergetic effect on vacancy formation is also studied. The present theoretical results show that the vacancy formation 
energy is significantly decreased when a $\mathrm{Na}$ atom and a $\mathrm{O}$ atom are co-adsorbed on the Mo (110) surface. Therefore, it can be inferred that impurity can facilitate the dissolution of Mo atoms and lead to corrosion. For the $\mathrm{Na} / \mathrm{O}$ co-adsorption condition, the existence of $\mathrm{Re}$ atom can increase the surface vacancy formation energy to $1.06 \mathrm{eV}$. Hence, Re can prevent the Mo alloy from corrosion in liquid Na with O impurities.

Table 5. Mo vacancy formation energy on different surface models.

\begin{tabular}{|c|c|c|}
\hline Adsorbate & Surface & Vacancy Formation Energy (eV) \\
\hline \multirow{2}{*}{ Clean } & Mo (110) & 1.45 \\
\hline & Mo-Re (110) & 1.32 \\
\hline \multirow{2}{*}{ A single $\mathrm{O}$ atom } & Mo (110) & 0.97 \\
\hline & Mo-Re (110) & 0.86 \\
\hline \multirow{2}{*}{ A single $\mathrm{Na}$ atom } & Mo (110) & 0.93 \\
\hline & Mo-Re (110) & 0.90 \\
\hline \multirow{2}{*}{$\mathrm{Na}$ and $\mathrm{O}$ co-adsorption } & Mo (110) & 0.47 \\
\hline & Mo-Re (110) & 1.06 \\
\hline
\end{tabular}

\section{Conclusions}

In this study, the adsorption and diffusion behaviors of $\mathrm{Na}$ and $\mathrm{O}$ atoms on BCC-Mo (110) surface are investigated by a first-principles approach. It is found that the Hollow site is the most energetically preferred adsorption site for a single $\mathrm{Na}$ or $\mathrm{O}$ atom. The existence of a Re alloy atom in the first atomic layer of the surface can strengthen the attractive interaction between the adsorbate and the substrate. The diffusion barrier of the $\mathrm{Na}$ atom on the Mo (110) surface is only $0.037 \mathrm{eV}$, and the pre-adsorbed $\mathrm{O}$ atom and Re atom can significantly impede Na diffusion. The surface vacancy formation energy is calculated for evaluating the stability of the Mo (110) surface. It is found that the Na or O atom can decrease the formation energy of the surface vacancy. It is worth noting that the $\mathrm{Na} / \mathrm{O}$ co-adsorption can significantly reduce the vacancy formation energy to $0.47 \mathrm{eV}$ for the $\mathrm{Mo}$ (110) surface, which indicates that the dissolution of surface atoms is a potential mechanism for the Mo in the Na liquid with $\mathrm{O}$ impurity. However, Re as alloy element can increase the resistance to the dissolution induced by $\mathrm{Na} / \mathrm{O}$ co-adsorption.

Author Contributions: Q.Z. performs simulation and data analyzation, and writes the original draft; Z.L. concept this work; W.L., M.M. and H.D. refine the manuscript. All authors have read and agreed to the published version of the manuscript.

Funding: This study is financially supported by the President Foundation of China Academy of Engineering Physics (YZJJLX2018002).

Institutional Review Board Statement: Not applicable.

Informed Consent Statement: Not applicable.

Data Availability Statement: Data sharing is not applicable to this article.

Conflicts of Interest: The authors declare no conflict of interest.

\section{References}

1. Zhang, H.; Zhuang, J. Research, development and industrial application of heat pipe technology in China. Appl. Therm. Eng. 2003, 23, 1067-1083. [CrossRef]

2. Ai, B.; Chen, S.; Yu, J.; Lu, Q.; Han, H.; Hu, L. Fabrication of lithium/C-103 alloy heat pipes for sharp leading edge cooling. Heat Mass Transf. 2017, 54, 1359-1366. [CrossRef]

3. Wang, C.; Guo, Z.; Zhang, D.; Qiu, S.; Tian, W.; Wu, Y.; Su, G. Transient behavior of the sodium-potassium alloy heat pipe in passive residual heat removal system of molten salt reactor. Prog. Nucl. Energy 2013, 68, 142-152. [CrossRef]

4. Faghri, A. Review and Advances in Heat Pipe Science and Technology. J. Heat Transf. 2012, 134, 123001. [CrossRef]

5. Reay, D.; McGlen, R.; Kew, P. Heat Pipes: Theory, Design and Applications, 6th ed.; Elsevier: Amsterdam, The Netherlands, 2014.

6. Jouhara, H.; Chauhan, A.; Nannou, T.; Almahmoud, S.; Delpech, B.; Wrobel, L. Heat pipe based systems-Advances and applications. Energy 2017, 128, 729-754. [CrossRef] 
7. Qu, W. Progress Works of High and Super High Temperature Heat Pipes. In Developments in Heat Transfer; Bernardes, M.A.D.S., Ed.; InTech: London, UK, 2011; pp. 503-522. ISBN 978-953-307-569-3.

8. El-Genk, M.; Tournier, J.-M. Challenges and fundamentals of modeling heat pipes' startup from a frozen state. AIP Conf. Proc. 2002, 608, 127-138.

9. Lundberg, L.B. Refractory Metals in Space Nuclear Power. JOM 1985, 37, 44-47. [CrossRef]

10. Lundberg, L. An evaluation of molybdenum and its alloys. In Proceedings of the 16th Thermophysics Conference, American Institute of Aeronautics and Astronautics (AIAA), Palo Alto, CA, USA, 23-25 June 1981.

11. King, J.C.; El-Genk, M.S. Review of Refractory Materials for Alkali Metal Thermal-to-Electric Conversion Cells. J. Propuls. Power 2001, 17, 547-556. [CrossRef]

12. El-Genk, M.S.; Tournier, J.-M. A review of refractory metal alloys and mechanically alloyed-oxide dispersion strengthened steels for space nuclear power systems. J. Nucl. Mater. 2005, 340, 93-112. [CrossRef]

13. Hampel, C. Refractory Metals. Tantalum, Niobium, Molybdenum, Rhenium, and Tungsten. Ind. Eng. Chem. 1961, 53, 90-96. [CrossRef]

14. Shmelev, A.N.; Kozhahmet, B.K. Use of molybdenum as a structural material of fuel elements for improving the safety of nuclear reactors. J. Phys. Conf. Ser. 2017, 781, 012022. [CrossRef]

15. Gilbert, M.; Packer, L.; Stainer, T. Experimental validation of inventory simulations on molybdenum and its isotopes for fusion applications. Nucl. Fusion 2020, 60, 106022. [CrossRef]

16. Lundberg, L. Critical Evaluation of Molybdenum and Its Alloys for Use in Space Reactor Core Heat Pipes; No. LA-8685-MS; Los Alamos Scientific Laboratory: Los Alamos, NM, USA, 1981. [CrossRef]

17. Yu, X.; Kumar, K. Uniaxial, load-controlled cyclic deformation of recrystallized molybdenum sheet. Mater. Sci. Eng. A 2012, 540, 187-197. [CrossRef]

18. Jörg, T.; Music, D.; Hauser, F.; Cordill, M.J.; Franz, R.; Köstenbauer, H.; Winkler, J.; Schneider, J.; Mitterer, C. Deformation behavior of Re alloyed Mo thin films on flexible substrates: In situ fragmentation analysis supported by first-principles calculations. Sci. Rep. 2017, 7, 7374. [CrossRef]

19. Agnew, S.R.; Leonhardt, T. The low-temperature mechanical behavior of molybdenum-rhenium. JOM 2003, 55, 25-29. [CrossRef]

20. DiStefano, J.R. Review of alkali metal and refractory alloy compatibility for Rankine cycle applications. J. Mater. Eng. 1989, 11, 215-225. [CrossRef]

21. Tu, S.-T.; Zhang, H.; Zhou, W.W. Corrosion failures of high temperature heat pipes. Eng. Fail. Anal. 1999, 6, 363-370. [CrossRef]

22. Kresse, G.; Furthmüller, J. Efficiency of ab-initio total energy calculations for metals and semiconductors using a plane-wave basis set. Comput. Mater. Sci. 1996, 6, 15-50. [CrossRef]

23. Kresse, G.; Furthmüller, J. Efficient Iterative Schemes for Ab Initio Total-Energy Calculations Using a Plane-wave Basis Set. Phys. Rev. B 1996, 54, 11169-11186. [CrossRef]

24. Car, R.; Parrinello, M. Unified Approach for Molecular Dynamics and Density-Functional Theory. Phys. Rev. Lett. 1985, 55, 2471-2474. [CrossRef]

25. Jones, R.O.; Gunnarsson, O. The density functional formalism, its applications and prospects. Rev. Mod. Phys. 1989, 61, 689-746. [CrossRef]

26. Payne, M.C.; Teter, M.P.; Allan, D.C.; Arias, T.A.; Joannopoulos, A.J. Iterative minimization techniques for ab initio total-energy calculations: Molecular dynamics and conjugate gradients. J. Rev. Mod. Phys. 1992, 64, 1045. [CrossRef]

27. Blöchl, P.E. Projector augmented-wave method. Phys. Rev. B 1994, 50, 17953-17979. [CrossRef] [PubMed]

28. Kresse, G.; Joubert, D. From ultrasoft pseudopotentials to the projector augmented-wave method. Phys. Rev. B 1999, 59, 1758. [CrossRef]

29. Perdew, J.P.; Burke, K.; Ernzerhof, M. Generalized Gradient Approximation Made Simple. Phys. Rev. Lett. 1996, 77, 3865. [CrossRef]

30. Monkhorst, H.J.; Pack, J.D. Special points for Brillouin-zone integrations. Phys. Rev. B 1976, 13, 5188-5192. [CrossRef]

31. Jain, A.; Ong, S.P.; Hautier, G.; Chen, W.; Richards, W.D.; Dacek, S.; Cholia, S.; Gunter, D.; Skinner, D.; Ceder, G.; et al. Commentary: The Materials Project: A materials genome approach to accelerating materials innovation. APL Mater. 2013, 1, 011002. [CrossRef]

32. Okamoto, H. Supplemental Literature Review of Binary Phase Diagrams: Ag-Yb, Al-Co, Al-I, Co-Cr, Cs-Te, In-Sr, Mg-Tl, Mn-Pd, Mo-O, Mo-Re, Ni-Os, and V-Zr. J. Phase Equilibria Diffus. 2016, 37, 726-737. [CrossRef]

33. Henkelman, G.; Uberuaga, B.; Jónsson, H. A climbing image nudged elastic band method for finding saddle points and minimum energy paths. J. Chem. Phys. 2000, 113, 9901-9904. [CrossRef]

34. American Society for Metals and ASM Handbook Committee. Properties and Selection: Nonferrous Alloys and Pure Metals, 9th ed.; American Society for Metals: Russell Township, OH, USA, 1979.

35. Guo, F.; Wang, J.; Du, Y.; Wang, J.; Shang, S.-L.; Li, S.; Chen, L. First-principles study of adsorption and diffusion of oxygen on surfaces of TiN, ZrN and HfN. Appl. Surf. Sci. 2018, 452, 457-462. [CrossRef]

36. Graciani, J.; Sanz, J.F.; Asaki, T.; Nakamura, K.; Rodriguez, J.A. Interaction of oxygen with $\mathrm{TiN}(001): \mathrm{N} \leftrightarrow \mathrm{O}$ exchange and oxidation process. J. Chem. Phys. 2007, 126, 244713. [CrossRef] [PubMed]

37. Osei-Agyemang, E.; Balasubramanian, G. Surface oxidation mechanism of a refractory high-entropy alloy. Npj Mater. Degrad. 2019, 3, 20. [CrossRef] 